\title{
Efeito da infecção hospitalar da corrente sanguínea por Staphylococcus aureus resistente à oxacilina sobre a letalidade e o tempo de hospitalização
}

\author{
M. Moreira, E.A.S. Medeiros, A.C.C. Pignatari, S.B. Wey, D.M. Cardo
}

Serviço de Controle e Prevenção de I nfecção Hospitalar e Disciplina de Doenças I nfecciosas e Parasitárias - Universidade F ederal de São Paulo, São Paulo, SP.

RESUMO - Oвj etivo. Determinar a letalidade atribuída à infecção hospitalar da corrente sangüínea (IHCS) por Staphylococcus aureus resistente à oxacilina (SARO) e seu efeito sobre o tempo de hospitalização.

Casuística e Métodos. Estudo tipo caso controle envolvendo 71 pares de pacientes adultos internados em hospital de ensino no período de janeiro de 1991 a setembro de 1992, pareados para os seguintes critérios: idade, sexo, doença de base, procedimento cirúrgico, mesmo período de risco e data de admissão.

Resultados. A incidência de pacientes com sepses hospitalar por SARO representou $73,22 \%$ entre aqueles que desenvolveram bacteremia por Staphylococcus aureus. A taxa de letalidade dos casos foi de $56,33 \%$ (40/71). Oito controles morreram, o

\section{INTRODUÇÃO}

$\mathrm{Na}$ última década os microorganismos grampositivos, em especial o Staphylococcus aureus, emergiram como importantes agentes causadores de infecção da corrente sangüínea ${ }^{1-4}$. E stas infecções acometem pacientes em todas as faixas etárias, com mai or freqüência nos extremos de idade e apresentam pior prognóstico em pacientes com idade aci ma de 50 anos $^{5-10}$. Entre as infecções hospitalares, as sepses por Staphylococcus aureus são responsáveis por el evada morbidade e mortalidade ${ }^{8,10,11}$.

As primeiras cepas de Staphylococcus aureus resistente à oxacilina (SARO) foram descritas na década de $60^{12,13}$ porém este microorganismo tornou-se importante causa de infecção hospitalar no início da década de 70, quando começaram a ser descritos os primeiros surtos ${ }^{14,15}$. Depois de 1975, o SARO tornou-se problema nos Estados Unidos da América, com aumento no número de surtos em várias instituições, 16,17 e hoje são endêmicos em muitos hospitais ${ }^{18-25}$, contribuindo, inclusive, com o aumento das taxas de infecção hospitalar ${ }^{25}$.

No Brasil, o SARO é responsável por 26,6 a $71 \%$ das cepas de Staphylococcus aureus isoladas em diversos hospitais do país ${ }^{26-29}$. Sader et al., em 1994, identificaram uma cepa de SARO de origem comum entre diversas instituições brasileiras, que corresponde à taxa de letalidade de $11,26 \%$ (8/71). A letalidade atribuída à infecção hospitalar da corrente sangüínea por SARO foi de 45,07\% (OR =17,0; IC 95\%=3,58 - 202,26; $p=0,000001$ ). Os casos permaneceram, em média, 32,5 dias internados no hospital, enquanto que os controles 29,7 dias $(p=0,32)$.

Conclusões. Observou-se elevada proporção de sepses por SARO entre todas as bacteremias por Staphylococcus aureus. A IHCS por SARO acarreta, por si só, uma alta taxa de letalidade, independentemente da doença que causou a internação, sem contudo, aumentar o tempo de permanência hospitalar.

UNITERMOS - Sepses. Mortalidade. Staphylococcus aureus. Infecção hospitalar.

sugerindo que exista transmissão interhospitalar desse microorganismo.

Relatos do Center for Disease Control and Pre vention (CDC) de 1982 sugeriam que as infecções por SARO envolviam, predominantemente, grandes centros terciários de referência e as instituições universitárias ${ }^{30}$. Porém, dados mais recentes, mostram que $96 \%$ dos hospitais nos EUA que fazem vigilância epidemiológica, tiveram pacientes com infecção por SARO no período de 1987 a $1989^{24}$.

Sabemos que as infecções por SARO apresentam alta letalidade, variando entre 4,5 a $50 \%^{24,31-35}$ e se considerarmos especificamente as bacteremias, estas variam de 5 a $47 \%$, dependendo das unidades estudadas e do tratamento instituído $35-44$.

Atualmente, a incidência de infecções por este microorganismo continua alta, e estudos vêem demostrando que o SARO apresenta letalidade maior que os Staphylococcus aureus sensível à oxacilina (OSSA)45,46. Romero-Vivas et al., em 1995, demostraram através de um estudo que houve relação entre mortalidade e resistência à oxacilina.

Conterno, em 1994, no Hospital São Paulo, Brasil, demonstrou que o Staphylococcus aureus foi um agente importante das bacteremias, principalmente hospitalares, que a letalidade foi el evada e que o SARO foi identificado como fator de risco independente para a letalidade. 
Poucos trabalhos têm avaliado o efeito da IHCS por SARO sobre a letalidade e o tempo de hospitalização.

\section{CASUÍSTICA E MÉtodos}

\section{Hospital São Paulo}

O Hospital São Paulo (HSP), instituição de ensino da Universidade Federal de São Paulo (UNIFESP), tem aproximadamente 660 leitos, distribuídos em 25 enfermarias de diversas especial idades e presta assistência médica à população local, de outros municípios do estado e do país. Nos anos de 1991 e 1992 ocorreram 15.404 internações nas unidades estudadas do HSP, com um coeficiente de letalidade de $8.99 \%$.

\section{Planejamento do Estudo}

Os casos foram obtidos a partir de revisão de relatórios mensais do Laboratório Central e da vigilância epidemiológica das infecções hospitalares realizada prospectivamente pelo Serviço de Prevenção e Controle de Infecção Hospitalar do HSP-UNIFESP, no período de janeiro de 1991 a setembro de 1992. Em posterior revisão de prontuários, obtivemos 93 pacientes com infecção hospitalar da corrente sangüínea (IHCS) por SARO, avaliados pelo antibiograma e internados em diversos setores do HSP, como as unidades de terapia intensiva, nefrologia, hematologia e outras unidades cirúrgicas e clínicas. Definiu-se como infecção hospitalar aquela que não estava presente no momento da internação, ou que apresentasse evi dência de estar ligada a procedimento terapêutico como hemodiálise, diálise peritoneal, quimioterapia entre outros ${ }^{47}$. Considerou-se hospitalar quando a hemocultura obtida, após 48 hs de internação, apresentou crescimento de microorganismo ${ }^{48}$.

Foram excluídos do estudo dois pacientes que vieram transferidos de outro hospital, e dois por apresentarem falsa bacteremia. Este quadro foi definido como a presença de hemocultura positiva na ausência de sinais ou sintomas de infecção ${ }^{48}$, ou ainda, quando o paciente ficou curado sem uso de antibiótico e, ainda, quatro pacientes que não estiveram internados na ocasião da bacteremia. No final da avaliação foram incluídos no estudo 85 pacientes.

Foi definido como caso o paciente adulto com pel o menos uma hemocul tura positiva para SARO que ti vesse apresentado tratamento com um gl icopeptídeo (vancomicina ou teicoplanina) e evidência de sepses, definida pela presença de dois ou mais dos seguintes sintomas ou sinais: temperatura axilar maior que $38^{\circ} \mathrm{C}$ ou menor que $36^{\circ} \mathrm{C}$; freqüência cardíaca maior que 90 batimentos por minuto; freqüência respiratória maior que 20 incursões por minuto, $\mathrm{PaCO}_{2}$ menor que $32 \mathrm{mmH} \mathrm{g}$, hemograma com contagem de leucócitos superior a 12000 ou inferior a $4000 \mathrm{~mm}^{3}$.
Foi definido como controle o paciente internado no HSP-UNIFESP que não tivesse apresentado bacteremia para SARO durante o período de internação. Foram excluídos deste grupo pacientes com cultura positiva por SARO em qualquer outro sítio, sem relato de hemocultura negativa e os que foram tratados com vancomi cina ou quinol ona empiricamente por período maior de 24 hs. Os controles foram pareados para os seguintes critérios: mesmo sexo; diferença de idade não superior a 15 anos; similar diagnóstico na admissão hospitalar; similar diagnóstico secundário; similar procedimento cirúrgico; data de internação no hospital com diferença não superior a dois anos e similar período de risco definido como período de permanência hospitalar, em dias, igual ou superior ao período compreendido entre a admissão do paciente (caso) e o aparecimento da bacteremia pelo SARO.

\section{Métodos Laboratoriais}

A col eta de sangue para hemocul tura foi realizada pela equipe de enfermagem, seguindo uma rotina que não foi alterada durante o período estudado.

Foi utilizado frasco contendo meio de Triplic Soy Broth - TSB (DIFCO) e placas de ágar sangue e os germes isolados identificados segundo as técnicas clássicas ${ }^{49}$.

Utilizou-se a técnica Bauer e Kirby de difusão em disco na determinação da resistência à drogas antimicrobianas ${ }^{50}$. O disco era impregnado com oxacilina na concentração de $1 \mu \mathrm{g} /$ disco e a resistência à oxacilina definida com um diâmetro de hal o de inibição de crescimento igual ou inferior a $10 \mathrm{~mm}$.

\section{Análise Estatística}

Para testarmos a hipótese de que a letalidade no grupo de pacientes com IHCS por SARO foi estatisticamente significante aos controles, foi utilizado o teste de $\mathrm{McNemar}{ }^{51}$. A duração da hospitalização dos pacientes para caso e controles foi estabelecida pelo teste de Wilcoxon ${ }^{52}$.

\section{RESULTADOS}

No período de janeiro de 1991 a setembro de 1992 foram diagnosticados 127 pacientes com I HCS por Staphylococcus aureus, e, destes, 93 (73,22\%) por SARO. Excluindo os pacientes não internados, aqueles com falsa bacteremia e transferidos de outro hospital, foram analisados 85 pacientes.

Foi possível encontrar um controle para 71 dos 85 pacientes (83,52\%). Os 14 casos restantes apresentavam diagnóstico pouco comuns, ou período prol ongado entre a internação e o apar eci mento da bacteremia, fatos que dificultaram o encontro dos controles.

\section{Incidência}

Dos 85 pacientes que apresentavam IHCS por SARO, 25 adquiriram a infecção quando interna- 
Tabela 1 - Tempo (dias) de aparecimento da infecção hospitalar da corrente sangüínea por Staphylococcus aureus resistente à oxacilina nos 71 casos internados no Hospital São Paulo - UNIFESP, no período de janeiro de 1991 a setembro de 1992

\begin{tabular}{|lcc|}
\hline Tempo (dias) & \multicolumn{2}{c|}{ Casos } \\
& Número & Porcentagem \\
0 -------- 2 & 10 & 14,08 \\
3 ------ 10 & 16 & 22,53 \\
11 ----- 20 & 22 & 30,98 \\
21 dias e mais & 23 & 32,39 \\
\hline
\end{tabular}

Tabela 2-Sucesso das variáveis pareadas dos pacientes com infecção hospitalar da corrente sangüínea por Staphylococcus aureus resistente à oxacilina, casos e seus controles, internados no Hospital São Paulo - UNIFESP, no período de janeiro de 1990 a dezembro de 1993.

\begin{tabular}{lccc|} 
Variáveis & $\begin{array}{r}\text { No total } \\
\text { de pares }\end{array}$ & $\begin{array}{c}\text { Número } \\
\text { atingido }\end{array}$ & $\begin{array}{c}\text { Porcentagem } \\
\text { de sucesso }\end{array}$ \\
Idade* & 71 & 63 & 88,73 \\
Sexo & 71 & 65 & 91,54 \\
Data de admissão & 71 & 69 & 97,18 \\
Similar período de risco & 71 & 66 & 92,95 \\
Diagnóstico de admissão** & 71 & 71 & 100,00 \\
Similar procedimento cirúrgico & 31 & 26 & 83,87 \\
Total & 386 & 359 & 93,00 \\
\hline Fonte: Prontuários Médicos, HSP. \\
* Idade com diferença não superior a 15 anos \\
** Data de admissão com diferença não superior a 2 anos
\end{tabular}

dos em UTI, 20 pacientes em unidades cirúrgicas e 40 em unidades clínicas.

\section{Tempo de aparecimento da infecção}

Observamos uma média de 16,6 dias de internação para o aparecimento da infecção. Sendo que 63,38\% dos pacientes apresentaram a infecção após 11 dias de internação.

Obtivemos um sucesso no pareamento entre casos e controles de $83,52 \%$ ou seja, foi possível encontrar um controle para 71 dos 85 pacientes; das variáveis utilizadas para o pareamento, houve uma taxa de sucesso de $93 \%$, sendo que o diagnóstico de admissão alcançou sucesso de pareamento de $100 \%$ dos casos.

\section{Letalidade}

A taxa de letalidade dos casos foi de $56,33 \%$ (40 dos 71 casos morreram). Oito controles morreram, o que corresponde a taxa de letalidade de $11,26 \%$. A letalidade atribuída à infecção hospitalar da corrente sangüínea por SARO foi de 45,07\% ("Odds Ratio" = 17,0; I ntervalo de Confiança de 95\% =3,58 - 202,26; $p=0,000001$ ). Trinta e quatro casos morreram, enquanto os seus respectivos controles sobreviveram (tabela 3).
Tabela 3 - Letalidade dos 71 pares de pacientes, casos com infecção hospitalar da corrente sanguínea por Staphylococcus aureus resistente à oxacilina e seus respectivos controles, internados no Hospital São Paulo - UNIFESP, no período de janeiro de 1991 a setembro de 1992.

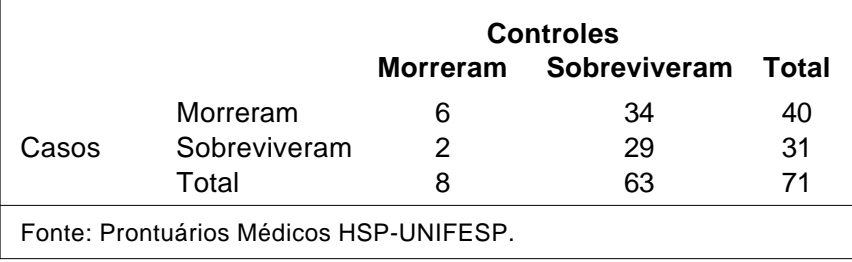

Tabela 4 - Média, mediana, desvio padrão, tempo mínimo e máximo de permanência em dias no Hospital São Paulo, de pacientes casos com infeccão hospitalar da corrente sanguínea por Staphylococcus aureus resistente à oxacilina e seus controles.

\begin{tabular}{lcc|}
\hline Estatística & Caso & Controle \\
Média & 32,55 & 29,75 \\
Mediana & 26 & 27 \\
Desvio padrão & 24,42 & 20,15 \\
Tempo mínimo & 3 & 6 \\
Tempo máximo & 139 & 128 \\
\hline
\end{tabular}

\section{Tempo de Permanência Hospitalar}

Os casos permaneceram em média 32,5 dias internados no hospital, enquanto que os controles $29,7(p=0,32)$.

\section{DISCUSSÃO}

As infecções hospital ares da corrente sangüínea vêm apresentando um aumento na incidência em diversas regiões do mundo, principalmente em grandes hospitais e nos universitários ${ }^{53}$.

As bactérias gram-positivas, especialmente o SARO, vêm contribuindo significativamente para o aumento das taxas de infecção hospitalar $16,21,22,23,25,54,65,66$

Em nosso estudo, o SARO representou aproximadamente $70 \%$ das infecções hospitalares da corrente sangüínea por Staphylococcus aureus. Esta incidência é considerada alta, quando comparada à estudos prévios, mesmo sendo o HSP - UNIFESP uma instituição de ensino de grande porte. Provavel mente, deve-se ao fato da grande utilização de anti bióticos, ea não-adoção de medi das específicas de controle para SARO, por serem onerosas e difíceis de implantar.

Em nosso meio, os hospitais universitários de grande porte funcionam como centros de referências terciárias, recebendo grande número de pacientes transferidos com doenças graves, necessitando, muitas vezes, de procedimentos invasivos ou permanecendo em unidades de terapia intensiva, o 
que facilita a disseminação do SARO, e portanto, aumentando a inci dência de infeção hospitalar por esse agente $\mathrm{e}^{55}$.

Quando analisamos estudos específicos sobre bacteremia por Staphylococcus aureus encontramos que de 30 a $50 \%$ dessas infecções da corrente sangüínea ocorrem por SARO $31,43,56,57$.

$\mathrm{E} \mathrm{m}$ nosso estudo, todas as infecções estudadas por SARO foram hospitalares, dado este concernente ao encontrado por outros autores ${ }^{19,43}$.

No período de aquisição da infecção, $29,41 \%$ dos pacientes estavam internados em unidade de terapia intensiva (UTI), mostrando a alta incidência deste agente nestas unidades, quer na vigência de surtos ou de forma endêmi ca ${ }^{66-69}$.

A média do tempo de aparecimento da sepses hospitalar por SARO foi de 16,6 dias; 45 pacientes $^{63,38}$ adquiriram a infecção após 11 dias de internação. Observa-se aumento após este período, pois geral mente os pacientes começam a apresentar complicações, necessitando ser submeti dos à procedimentos diagnósticos e terapêuticos, que predispõem ao aparecimento da infecção. Os pacientes que adquiriram infecção precoce, entre 0 e 2 dias de hospitalização, vinham sendo submetidos à procedimentos hospitalares em regime ambulatorial. Um dos fatores de risco importante para a aquisição de infeção por SARO é o tempo prol ongado de internação $22,31,37,46,58,59,60$.

Não encontramos qualquer estudo controlado que tenha avaliado a letalidade atribuída e o tempo de hospitalização da sepses hospitalar por SARO. Estudos controlados para a análise epidemiológica das infecções hospitalares são fundamentais para se estabelecer os reais efeitos dessas infecções sobre a evolução dos doentes. O pareamento tem a finalidade de reduzir a interferência de variáveis confundidoras, que poderiam influir na evolução do paciente.

Procuramos aproximar, em semelhança ao máximo casos e control es, sendo que dos 85 pacientes internados nas diversas unidades do hospital chegamos aos 71 melhores controles e não encontramos controle adequado para 14 pacientes. Assim, $79,77 \%$ da população que desenvolveu IHCS por SARO foi analisada.

Das 386 variáveis possíveis de pareamento, obtivemos sucesso em 359 (93\%), o que leva a concluir que expostos e não-expostos são semel hantes no conjunto. O pareamento atingiu acima de $90 \%$ de sucesso nos critérios sexo, data de admissão, (com diferença não superior a 2 anos) e igual período de risco. O diagnóstico de admissão atingiu pareamento de $100 \%$, sem contudo ter sido feito uma avaliação detalhada da gravidade da doença. Atingimos um sucesso de $88,73 \%$ no pareamento quanto à idade, sendo que a média das idades dos casos foi de 49,1 anos e dos control es foi de 48,5 anos, mostrando uma homogeneidade entre os grupos.
A letalidade no grupo de casos foi de $56,33 \%$ e no grupo controle de $11,26 \%$. A letalidade atribuída a IHCS por SARO foi de 45,07\%, "Odds ratio" de 17,0, I nterval o de Confiança de 95\% ( 3,58 - 202,26; $p=0,000001$ ). I sto significa que a sepses adquirida durante a hospitalização traz em si mesma maior letalidade, independentemente das condições de base. O paciente com sepses hospitalar por SARO tem aumentado em até 17,0 vezes o risco de morte devido ao efeito dessa infecção.

Esta letalidade atribuída à infecção hospitalar da corrente sangüínea por SARO foi a maior das descritas anteriormente para outros agentes, ${ }^{61-64}$ sugerindo ser o microrganismo de alta virulência. Não estudamos detalhadamente a concomitância da infecção em outros sítios como pul mão, coração, sistema nervoso central como porta de entrada ou complicação da sepses, mas sabe-se que nestas, a letalidade costuma ser maior que nas infecções cuja porta de entrada é o catéter venoso central .

Dados recentes mostram que as bacteremias por SARO apresentam características diferentes daquel as por OSSA e em anál ise estatística multivariada houve rel ação entre a mortali dade e a resistência a methicillina ${ }^{44,46}$.

Ao analisarmos o tempo de permanência hospitalar, observamos que os casos permaneciam em média 32,5 dias, enquanto os controles 29,7 dias, não ocorrendo, portanto, aumento no tempo de internação exclusivamente atribuída a infecção hospitalar da corrente sangüínea por SARO.

Como o evento morte inter rompe a internação, analisamos posteriormente o grupo de pares em que ambos, casos e controles, sobreviveram e mesmo com esta análise não notamos aumento estatisticamente significante no período de permanência hospitalar dos casos. Provavelmente este fato ocorreu pois o pareamento foi muito rigoroso, ou seja, a escol ha de controles com doença de base tão grave quanto aos casos implicou em maior número de complicações não infecciosas nestes pacientes, com prolongamento no tempo de internação.

Com este trabalho pudemos concluir que em nossa instituição existe elevada proporção de SARO dentre as bacteremias por Staphylococcus aureus e que esta acarreta, por si só, uma al ta taxa de letalidade, mostrando a necessi dade de estudos para a implantação de medidas de controle desse microorganismo.

\section{SUMMARY}

\section{The effect of bloodstream hospital infection by Staphylococcus aureus resistant to methi- cillin on the mortality and the length of hos- pitalization}

OBJ ECTIVES. To identify the attributed mortality rate of bloodstream hospital infection by Staphylococcus aureus resistant to methicillin (MRSA) and its effect on length of hospital stay. 
Disign.Casecontrol study

Setting. Hospital São Paulo da Universidade Federal de São Paulo, a 660-bed, tertiary-care teaching hospital in São Paulo, Brazil.

PATIENTS. Seventy one adults patients with hospital-acquired MRSA bacteremia diagnosed be tween J anuary 1, 1991, and September 30, 1992, and 71 MRSA-free controls were matched by the following criteria: age, sex, underlying disease, surgical procedure, samerisk timeand admissiom date.

RESULTS. The incidence of patients with hospital sepsis by MRSA accounted for $73.22 \%$ of the patients with hospital bloodstream infection by Staphylococcus aureus. The mortality rate of the cases was 56.33 (40/71) and 11.26 (8/71) of the controls. The attributable mortality rate was $45.07 \%$ (OR=17.0; I C 95\%=3.58 - 202.26; $p=0.000001)$. The length of hospital stay median time was of 32.55 days for the cases and 29.75 for the controls $(p=0.32)$.

ConcLusion. A high level of sepsis by MRSA was observed in all the Staphylococcus aureus bacteremia. The bloodstream hospital infection by MRSA itself does provide a high level of mortality independently from the patients base disease, without however, increasing their hospital length of stay. [Rev Ass Med Bras 1998; 44(4): 263-8.]

KEY WORDS: Sepsis. Mortality. Staphylococcus aureus. Cross infection.

\section{REFERÊNCIAS BIBLIOGRÁFICAS}

1. Salomão R, Castelo A, Pignatari ACC, Wey SB. Nosocomial and community acquired bacteremia:variables associated with outcomes. Rev Paul Med 1993; 111: 456-461.

2. CDC - Centers for DiseaseControl and Prevention - Nosocomial infections Surveillance/1980, 82. C.D.C. Surveillance Summaries 1983; 32: 155-65.

3. Morrison AJ J r, Freer CV, Searcy MA, Landry SM, Wenzel RP. Nosocomial bloodstream infections: secular trends in a statewide surveillance program in Virginia. Infect Control 1986; 7: 550-3.

4. MacGowan J E J r. Changing etiology of nosocomial bacteremia and fungemia and other hospital-acquired infections. ReV Infect Dis 1985; 7(suppl.3): S357-S370.

5. Shah M, Watanakunakorn C. Changing patterns of StaphyIococcus aureus bacteremia. AmJ Med Sci 1979; 278: 115-21.

6. Mirimanoff RO, Glauser MP. Endocarditis during Staphylococcus aureus septicemia in a population of non-drug addicts. Arch Intern Med 1982; 142: 1.311-13.

7. Bryan CS, Kirkhart B, Brenner ER. Staphylococcal bacteremia: current patterns in nonuniversity hospitals. South Med J 1984; 77: 693-6.

8. Gransden WR, Eykyn SJ , Phillips I. Staphylococcus aureus bacteremia: 400 episodes in St. Thomas's Hospital. Br Med J 1984; 288: 300-303.

9. Libman H, Arbeit RA. Complications associated with StaphyIococcus aureus bacteremia. Arch Inter Med 1984; 114: 541-5.

10. Watanakunakorn C, Chan SJ , Demargo DG, Palmer J A. Staphylococcus aureus bacteremia: significance of hyperbilirubinemia. Scand J Infect Dis 1987; 19: 195-203.

11. MylotteJ M,McDermott; Spooner J A. Prosrectivestudy of 114 consecutive episodes of Staphylococcus. Rev Infect Dis 1987; 9: 891-907.
12. J evons MP "Celbenim" Resistant Staphylococci. Br Med J $1961 ; 1: 124$.

13. Parker MT, Hewitt J G. Methicillin-resistant in Staphylococcus aureus. Lancet 1970; 1: 800-804.

14. Barret FF, McGehee RF, Finland M. Methicillin-resistant Staphylococcus aureus at Boston City Hospital: bacteriologic and epidemiologic observations. N EnglJ Med 1968; 279: 441-8.

15. O'toole RD, Drew WL, Dahlgren BJ , Beat HN. An outbreak of methicillin-resistant Staphylococcus aureus: infection- observations in hospital and nursing home. J AMA 1970; 213: 257-63.

16. Boyce J M, Causey WA. Increasing occurrence of methicillinresistant Staphylococcus aureus in the United States. Infect Control 1982; 3: 377-83.

17. Casewell MW. Epidemiology and control of the "modern" methicillin-resistant Staphylococcus aureus. J Hosp Infect 1986; 7:1-11.

18. Benner EJ , Kayser FH. Growing clinical significance of methicillin-resistant Staphylococcus aureus. Lancet 1968; 5: 740-5.

19. Kayser FH, Mark TM. Metlhicilin-resistant Staphylococci. AmJ Med Sci 1972; 264: 197-205.

20. Boyce J M, Landry M, Deetz TR, Dupont HL. Epidemiologic studies of an outbreak of nosocomial methicillin-resistant Staphylococcus aureus infections. Infect Control 1981; 2: 110-16.

21. Linneman CC, Mason M, Moore P, Korfhagen TR, Stanek J L. Methicillin-resistant Staphylococcusaureusexperiencein a general hospital over four years. AmJ Epidemiol 1982; 115: 941-50.

22. Thompson RL, Cabezudol, Wenzel RP. E pidemiology of nosocomial infections caused by methicillin-resistant StaphyIococcus aureus. Ann Intern Med 1982; 97: 309-317.

23. Opal SM, Mayer KH, Stemberg MJ , Blazek J E, Mikolch DJ , Dicknsheets L, Lyhte LW, Trudei RR, Musser J M. Frequent acquisition of multiplestrains of methicillin-resistant Staphylococcus aureus by heal thcareworkers in an epidemic hospital environment. Infect Control Hosp Epidemiol 1990; 11: 479-85.

24. Boyce J M. Patterns of methicillin-resistant Staphylococcus aureus prevalence. Infect Control Hosp Epidemiol 1991; 12: 79-82.

25. Stamm AM, Long MN, Belcher B. Higher overal nosocomial infection rate because of encreased attack rate of methicillinresistant Staphylococcus aureus. Am J Infect Control 1993; 21: 70-4.

26. Levy CE,M ontelli AC, Furtado J S et al. Resistência a drogas em cepas bacterianas de pacientes de serviços hospitalares: laboratório de referência do sistema COBA. Rev Microbiol 1991; 22: 21-7.

27. Nunes, M.R.C.M.; Magalhães, P.P.; Farias, L.M.; Lima, M.C. Staphylococcus aureus multi-resistente em infecções hospitalares no Hospital Getúlio Vargas - Teresina - Piauí. In: Congresso Brasileiro de Controle de Infecção Hospitalar R, Recife, 1994, p.82.

28. Rezende, E.M.; Starling, C.E.F.; Couto, B.R.G.M.; Riegg, E.; Prado, I. - Prevalência de Staphylococcus aureus multiresistenteem hospitais gerais deB el oH orizonte. In: Congresso BrasileirodeControledel nfecçãoH ospitalar, Recife, 1994. p. 83.

29. Costa J M, Ramos IB, Carvalho DJ ,Dias J r R. Análise da sensibilidade de Staphylococcus

aureus hospitalar aos antimicrobianos no período de 1988 a 1993. In: Congresso Brasileiro de Controle de I nfecção H ospitalar, 4, Recife, 1994. p.83.

30. Haley RW, Highrower AW, Khabbaz RF, Thornsberry C, Martone WJ , Allen J R, Hugher J M. The emergence of methicillin-resistant Staphylococcus aureus infections in United States hospitals: Possiblerole of thehousestaff-patient transfer circuit. An Inter Med 1982; 97: 297-308.

31. Locksley RM, Cohen ML, Quin TC, Tompkens LS, Coyle MB, KeriharaJ .M., Counts GW. Multiple-resistant Staphylococcus 
aureus: introduction transmission and evolution of nosocomiol infection. An intern Med 1982; 97: 317-24.

32. J ambon MF, Beuscat C, Meyron M, Roue R. et le Geiss. Infections graves à Staphylocoques dorés résistants à la meticilline. La Press Med 1993; 22: 909-13.

33. Pujol M, Peña C, Pallares R, Ayats J , Arisa J , Gudiol F. Risk factors for nosocomial bacteremia dueto methicillin-resistant Staphylococcus aureus. EurJ Clin Microbiol Infect Dis 1994; 13: $96-102$.

34. CoelloR, J iménezJ , García M, ArroyoP, Minguez D, Fernández C, Cruzet F, Gaspar C. Prospective study of infection, colonization and carriage of methicillin-resistant Staphylococcus aureus in an outbreak affecting 990 patients. Eur J Clin Microbiol Infect Dis 1994; 13: 74-81.

35. J erningan J A, Clemence MA, Stott GA, Tittus MG, Al exander $\mathrm{CH}$, Palumbo CN, Farr BM. Control of methicillin-resistant Staphylococcus aureus at a University Hospital: one decade later. Infect Control Hosp Epidemiol 1995; 16: 686-96.

36. Crossley R, Loesch D, L andesman B, Mead K, Chern M, Strat R. An outbreac of infection caused by strains of Staphylococcus aureus resistant to methicillin and aminoglycosides: I clinical studies. J Infect Dis 1978; 139: 273-9.

37. Peacock J E, Marsik FJ, Wenzel RP. Methicillin-resistant Staphylococcus aureus: introdution and spread within a hospital. Ann Inter Med 1980; 93: 532-62.

38. Grieble HG, Krause SL, Pappas SA, Diconstanzo MB. The prevalence of high-level methicillin resistance in multiply resistant hospital Staphylococci. Medicine 1981; 60: 62-9.

39. Linneman CC, Mason M, Moore P, Korfhagen TR, Stanek J L. Methicillin-resistant Staphylococcus aureus: experience in a general hospital over four years. AmJ Epidemiol 1982; 115: 941-50.

40. Sorrel TC, Packham DR, Shanker S, Folds M, Meinro R. Vancomicin therapy for methicillin-resistant Staphylococcus aureus. Ann Intern Med 1982; 97: 344-50.

41. Lewis E, Saravolatz LA. Comparison of methicillin-resistant and methicillin-sensitive Staphylococcus aureus bacteremia. Am J Infect Control 1985; 13: 109-14.

42. Hunt J L, Purdue GF, Tuggle DW. Morbidity and mortality of an endemic pathogen: methicillin-resistant Staphylococcus aureus. AmJ Surg 1988;156: 524-8.

43. Cheng AF, French GL. Methicillin-resistant Staphylococcus aureusbacteremiain Hong Kong.J Hosp Infect 1988;12: 91-101.

44. Conterno LO. Fatores de risco para letalidade da bacteremia por Staphylococcus aureus. São Paulo, 1994 Tese (mestrado) Escola Paulista de Medicina - UNIFESP. p96.

45. Asensio A, Guerrero A, Quereda C, Lizán M, Martinez-Ferrer M. Colonization an infection with methicillin-resistant Staphylococcus aureus: Associated factors and erradication. Infect Control Hosp Epidemiol 1996; 17: 20-8.

46. Romero-Vivas J , Rubio M, Fernandez C, Picazo J J . Mortality associated with nosocomial bacteremia due to methicillin-resistant Staphylococcus aureus. Clin Infect Dis 1995; 21: 1.417-23.

47. Garner J S, J arvis WR, Hemori J G, Horan TC, Hughes J M. CDC definitions for nosocomial infections. AmJ Infect Control 1988;16: 128-140.

48. Pittet D. Nosocomial bloodstrean infections. In: Wenzel RP. Prevention and control of nosocomial infection. 2 ed Baltimore, Willians \& Wilkins. 1993 p.512-555.

49. K Ioos WE \& J orgensen J H. Staphylococci. In: Lennette EH, Balows A, Hauseler W J, Shadomy HJ, Manual of clinical microbiology,.4 ed, Washington, American Society for Microbiology 1985 p.143-153.

50. Bauer AW, Kirby WMM, Sherris J C, Turck M. Antibiotic suscetibility testing by a standardized single disc method. Am J Clin Pathol 1966; 45: 493-6.
51. Fleiss J L. The analysis of data from matched samples. In: Statiscal methods for rates and proportions. 2nd ed. New York, J ohn Wiley and Sons, 1981, p.113-9.

52. Conover WJ .Practical nonparametricstatistics. Second Edition. New York, J ohn Wiley \& Sons, 1nc. 1980, p.363-4.

53. Banerjee SN, Emori TG, Culver DL, Gayner RP, J arvis WR, Horen T, Edwards J R, Tolson J , Henderson T, Martone WJ \& NNIS Secular trends in nosocomial primary bloodstream infection in the United States. 1989. Am J Med 1991; 91 (suppl.3b): S86-S89.

54. Boyce J M, White RL, Spruell EY. I mpact of meticillin-resistant Staphylococcus aureus on the incidence of nosomial Staphylococcal infections. J Infect Dis 1983; 148: 763.

55. Sader SS, Pignatari AC, RichardJ H, Ronald NJ . Evaluation of interhospital spread of methicillin - resistant Staphylococcus aureus in São Paulo, Brazil, using pulsed-field gel electrophoresis of chromosomal DNA. Infect Control Hosp Epidemiol 1994; 15: 320-3.

56. Keone CT, Cafferkey MT.Reemergence of methicillin-resistant Staphylococcus aureuscausing severeinfections. J Infect 1984; 9: 6-16.

57. Rinland D. A comparirison of methicillin-resistant (SARO) and methicillinsusceptible (OSSA) Staphylococcus aureus bacteremias. In: Annual Meeting Infection Disease Society 31‥ N ew Orleons/USA, 1993. Abstracts. IDSA annual meeting, 1993 p.154. (Abstract, 27a)

58. Crossley K, Landesman B, Zarke D. An outbreak of infection caused by strains of Staphylococcus aureusresistant to methicillin and aminoglycosides: II

epidemiologic studies. J Infect Dis 1979; 139: 280-7.

59. Rinland D. Nosocomial infections with methicillin and tobramycin resistant Staphylococcus aureus: implication of physiotherapy in hospital - wide dissemination. AmJ Med Sci 1985; 290: 91-7.

60. Boyce J M. Methicillin-resistant Staphylococcus: detection, epidemiology, and control measures. Infect Dis Clin North Am 1989; 3: 901-3.

61. Rose R, Hunting KJ , Townsend TR, Wenzel RP. Morbidity mortalidy and economics of hospital acquired bloodstream infections: a controlled study. South Med J $r$ 1977; 70: 1.267-9.

62. Spengler RF \& Grenough WB. Hospital cost and mortality attributed to nosocomial bacteremias. J AMA 1978; 240:2.455-8.

63. Wey SB, Mori M, Pjallei M A, Woolson RF, Wenzel RP. H ospital - acquired candidemia. Theatributablemortality and excess length of stay. Arch Intern Med 1988; 148: 2.642-5.

64. Landry SL, Kaiser DL, Wenzel RP. Hospital Stay and mortality attributed to nosocomial enterococcol bacteremia: A controlled study. Am J Infect Control 1989; 17: 323-9.

65. Struelens MJ, Mertens R. National survey of methicillinresistant Staphylococcus aureus in Belgian hospitals: Detction methods, prevalence trends and infection control measures. Eur J Clin Microbiol Infect Dis 1994; 13: 56-62.

66. Voss A, Milatovic D, Wallrauch-Schwarz C, Rosdahl VT, Braveny I. Methicillinresistant Staphylococcus aureus in Europe. Eur J Clin Microbiol Infect Dis 1994; 13: 50-5.

67. Witte W, Braulke Ch, Heuck D, Cuny Ch. Analysis of nosocomial outbreaks with multiply and methicillin-resistant Staphylococcus aureus (SARO) in Germany: implications for hospital hygiene. Infection 1994; 22(suppl.2): S128-S134.

68. Blumberg LH, Klugman KP. Control of methicillin-resistant Staphylococcus aureus bacteraemia in high-risk areas. Eur J Clin Microbiol Infect Dis 1994; 13: 82-5.

69. Valls V, Gómez-Herruz P,González-Palacios R, Cuadros J A,Romanyk J P,Ena J . Long- term efficacy of a program to control methicillin-resistant Staphilococcus aureus. Eur J Clin Microbiol Infect Dis 1994; 13: 90-5. 